\title{
Advances on GenIV structural and fuel materials and cross-cutting activities between fission and fusion
}

\author{
Lorenzo Malerba ${ }^{1, *}$, Pietro Agostini ${ }^{2}$, Marjorie Bertolus $^{3}$, Fabienne Delage ${ }^{3}$, Annelise Gallais-During ${ }^{3}$, \\ Christian Grisolia ${ }^{4}$, Karine Liger $^{5}$, and Pierre-François Giroux ${ }^{6}$ \\ ${ }^{1}$ División de Materiales de Interés Energético, CIEMAT, Avda. Complutense 40, 28040 Madrid, Spain \\ 2 Dipartimento Fusione e Sicurezza Nucleare, ENEA-FSN, Via Enrico Fermi 45, 00044 Frascati, Roma, Italy \\ ${ }^{3}$ CEA, DEN, DEC, Centre de Cadarache, 13108 St-Paul-lez-Durance, France \\ ${ }^{4}$ CEA, IRFM, 13108 Saint-Paul-lez-Durance, France \\ ${ }^{5}$ CEA, DEN, DTN, Centre de Cadarache, 13108 St-Paul-lez-Durance, France \\ ${ }^{6}$ DEN-Service de Recherches Métallurgiques Appliquées, CEA, Université Paris-Saclay, 91191 Gif-sur-Yvette, France
}

Received: 12 March 2019 / Accepted: 4 June 2019

\begin{abstract}
This paper describes six projects, most of which are part of the research portfolio of the EERA JPNM, devoted to qualification, modelling and development of structural and fuel materials for advanced and innovative nuclear systems, with also two examples of projects addressing issues of cross-cutting interest through fusion and fission. The main conclusion is that the benefit of the coordination under the umbrella of, in this case, the EERA JPNM, is clearly felt in terms of better alignment of national programmes and subsequent leveraging of institutional funding, to integrate Euratom support. Likewise, the benefit of addressing specific issues of common interest for fusion and fission is not only beneficial because of cross-fertilisation, but also because it allows more rational use of human and infrastructural resources, avoiding duplications.
\end{abstract}

\section{Introduction}

The deployment of Generation IV (GenIV) systems will ensure the full sustainability of nuclear fission energy. These systems are able to produce more fuel than they consume, offer $\sim 50 \%$ higher thermal efficiency and increased standards of passive safety than current reactors, while reducing significantly the volume and radiotoxicity (decay time $<1000$ years) of nuclear waste. This will guarantee low-carbon energy production for centuries through recycling, without additional mining, in a circular economy, thereby making nuclear energy both societally and economically attractive. However, materials will be exposed to high levels of temperature and irradiation, in contact with potentially aggressive non-aqueous coolants, and the target is to design reactors for 60-year operation. Similarly, fuel and fuel elements will need to be designed for high burnups, with the possibility of burning minor actinides. Therefore, the development, screening and qualification of structural and fuel materials that suitably perform and are affordable, are crucial to make GenIV reactors an industrial and commercial reality, with positive impacts on the economy, safety, waste and sustainability of nuclear energy.

\footnotetext{
* e-mail: lorenzo.malerba@ciemat.es
}

Thermo-nuclear fusion represents in the longer term a virtually inexhaustible source of energy with potentially very high standards of sustainability, efficiency and safety, thanks to the wide availability on earth of deuterium and lithium (from which tritium is selfproduced by nuclear reaction in the reactor itself), the inert nature of the reaction products, the high density of energy that the reaction can provide and the inherent safety of the system. The main wastes in fusion are activated structural materials. These are moreover expected to withstand unprecedentedly harsh conditions in terms of thermal shocks, radiation dose, and also exposure to high temperature and contact with coolants/ tritium breeders, the compatibility with which needs in some instances to be demonstrated. Despite the differences between GenIV fission and fusion, because of the extreme conditions expected in both systems several materials issues are in common. On the other hand, the main safety issue for fusion is represented by tritium management in terms of need to reduce inventory and avoid release. Solutions to this problem bear commonalities with fission.

In this framework of structural and fuel materials for GenIV and fission/fusion cross-cutting issues, the present paper will describe six projects, four of which are ongoing as part of H2020 and roughly half way through, namely 
GEMMA, ${ }^{1}$ INSPYRE,${ }^{2} \mathrm{M}^{4} \mathrm{~F}^{3}$ and TRANSAT,${ }^{4}$ while the remaining two, MatISSE ${ }^{5}$ and PELGRIMM, ${ }^{6}$ are now concluded and were part of FP7. Of these six projects, four (GEMMA, INSPYRE, M4F and MatISSE) are integrating part of the research portfolio of the Joint Programme on Nuclear Materials of the European Energy Research Alliance (EERA-JPNM) ${ }^{7,8}$, which will also be described.

\section{The Joint Programme on Nuclear Materials of the European Energy Research Alliance (EERA JPNM)}

EERA $^{7}$ was created in 2007 as the initiative of a number of European public research centres in order to join forces and coordinate efforts towards a low carbon energy economy in Europe. Since 2014 it is an international non-profit association (EERA AISBL). Currently, it brings together more than 250 organisations and coordinates the work of around 50,000 researchers from 30 countries, being Europe's largest energy research community.

EERA's official mission is to help streamline regional, national and European efforts, in order to deliver scientific and technical results from basic research to the demonstration phase (TRLs 2-5) and ensure efficient transfer to industry and market. EERA is the research pillar of the European Union's Strategic Energy Technology Plan (SET-Plan). ${ }^{9}$

EERA's members work together in, currently, 17 research joint programmes (JPs). These pursue research goals along shared agendas covering the whole range of low-carbon energy technologies, including social and economic aspects of the energy transition and addressing also the systemic nature of the transition to a zero-carbon society.

The EERA JPNM is one of the 17 EERA JPs, one of the two dealing with materials and the only one dealing with nuclear energy related activities. As such, the EERA JPNM acts as bridge and link, in research terms, between nuclear energy and other low carbon energy sources and systems. The reason for the focus on materials is the pivotal importance that these have in view of safety and sustainability of nuclear energy, as well as innovation in the energy field in general.

The objective of the EERA JPNM is to improve safety and sustainability of nuclear energy by focusing on materials aspects. This has two implications:

\footnotetext{
${ }^{1}$ http://www.eera-jpnm.eu/gemma/?q=jpnm\&sq $=$ sub6

${ }^{2}$ http://www.eera-jpnm.eu/inspyre/

3 http://www.h2020-m4f.eu/

${ }^{4}$ http://transat-h2020.eu/

${ }^{5}$ http://www.fp7-matisse.eu/

${ }^{6}$ https://cordis.europa.eu/project/rcn/101413/factsheet/en

7 https://www.eera-set.eu/

8 http://www.eera-jpnm.eu/

${ }^{9}$ https://ec.europa.eu/energy/en/topics/technology-andinnovation/strategic-energy-technology-plan
}

- Better knowledge of materials behaviour under operating conditions, to select the most suited materials and define safe design rules, especially allowing for radiation and temperature effects, while caring for compatibility with coolants.

- Development of advanced materials with superior capabilities, either through improved and advanced fabrication and processing methods, or adoption of new types of materials, in terms of resistance to high temperature, irradiation and aggressive environments.

Three grand challenges (GC) have been accordingly identified (JPNM Vision Paper ${ }^{10}$ ):

- GC1: Elaborate design correlations, assessment and test procedures for the structural and fuel materials that have been selected for the demonstrators under the service conditions expected.

- GC2: Develop physical models coupled to advanced microstructural characterization to achieve high-level understanding and predictive capability.

- GC3: Develop innovative materials solutions and fabrication processes of industrial application to achieve superior materials properties, to increase safety and improve efficiency and economy.

A Strategic Research Agenda (SRA) ${ }^{11}$ identifies the research lines to be pursued in the EU to ensure that suitable structural and fuel materials are available for the design, licensing, construction and safe long-term operation of GenIV nuclear systems, including an analysis of corollary aspects such as infrastructures, education and training, interaction with industry and international cooperation.

Currently, more than 50 organisations collaborate under the coordination of the EERA JPNM, by contributing to at least one of the six subprogrammes in which its activities are organized, devoted to qualification, modeling and development of structural and fuel materials.

One of the main instruments of implementation of the SRA of the EERA JPNM, in terms of alignment of research actions between the different organisations involved, are the so-called pilot projects. These are small projects of 3-4 year duration focused on precise topics that result from the convergence of research interests and lines of organisations from different EU member states. The Euratom-funded projects launched under the umbrella of the EERA JPNM, which are described in this paper, are the result of juxtaposing a number of JPNM pilot projects under a consistent framework. As such, these projects should not be looked as separate entities, but rather as different contributions towards the goals set out by the EERA JPNM SRA.

\section{PELlets versus GRanulates: Irradiation, Manufacturing \& Modelling (PELGRIMM)}

PELGRIMM investigated Minor Actinides (MA) bearing fuels, shaped as pellets and beads, for GenIV-Sodium Fast

\footnotetext{
${ }_{10}$ https://www.eera-set.eu/vision-paper-of-jp-nuclear-materials/

11 http://www.eera-jpnm.eu/?q=jpnm\&sq=nboard
} 
Reactor (SFR) systems. Both MA transmutation options were considered, namely: MA homogeneous recycle in driver fuels and MA heterogeneous recycle on $\mathrm{UO}_{2}$ fuels located in radial core blankets. The consortium included research laboratories, universities and industries, sharing their progress and achievements, and leveraging their skills, both experimental and in modeling and simulation, on the following topics: fuel fabrication and characterization, including behaviour under irradiation, of both pellet and sphere-packed loaded core design fuel, extended to safety performance pre-assessment from normal operating conditions to transients and severe accidents, to keep the link between fuel investigations and key issues of core physics.

Innovative irradiation tests and Post-Irradiation Examinations (PIE) performed within the project have widely improved the knowledge on Am-bearing fuel behavior under irradiation for both fuel types: MADriver Fuels (MADF) i.e. (U,Pu,MA) $\mathrm{O}_{2}$ and MABearing Blanket fuels (MABB) i.e. $(\mathrm{U}, \mathrm{MA}) \mathrm{O}_{2}$, in spherepac and pellet forms. Regarding the MADF concept, the PIEs of the semi-integral SPHERE irradiation showed that, for comparable irradiation conditions the behaviour of fuels that are shaped differently, were quite similar. The main difference is related to the presence of fuel-clad mechanical interaction for fuels shaped as pellets, apparently unobservable for spherepacked fuels. MABB developments got over the first stages of its qualification program with the PIE of the first separated-effect irradiation MARIOS and the first semi-integral irradiation MARINE. MARIOS PIE showed the (U,Am) $\mathrm{O}_{2}$ discs (i.e. MABB fuel) to be in relatively good shape after irradiation in the temperature range of $1000{ }^{\circ} \mathrm{C}-1300{ }^{\circ} \mathrm{C}$. Irrespective of fuel porosity and irradiation temperature, no significant swelling was measured (only tailored porosity disks were slightly densified), and all helium produced during irradiation was released, whereas the released fractions of $\mathrm{Kr}$ and $\mathrm{Xe}$ were strongly temperature dependent.

Different routes for MA-bearing fuel fabrication processes were investigated to look up for enhancements such as simplification, robustness, lower secondary waste streams,.... The Am-bearing fuel for MARINE, shaped as pellet and spherepac, were prepared by infiltration of americium nitrate solutions in porous $\mathrm{UO}_{2}$ precursor beads prepared by sol-gel gelation. In addition, an alternate route, involving micro-wave internal gelation was set up and a new dedicated equipment is currently available. Meanwhile, the adaptation of the WAR route to the synthesis of AmBB spherepacs and then pellets provided encouraging results: high densified pellets were obtained. By proving the feasibility of these diversified fuel synthesis flowsheets, PELGRIMM has enlarged prospects for Ambearing fuel investigations. Moreover, significant improvements were done in fuel performance codes thanks to the introduction of more phenomenological models, upgraded numerical technics, more accurate properties laws, etc. The resulting code Benchmark outcomes are promising: simulations of SPHERE, SUPERFACT and MARIOS irradiations provided were quite consistent with PIE results for most of the cases.
Finally, an optimized core design loaded with (U,Pu, Am) $\mathrm{O}_{2}$ spherepac driver fuels was calculated and its safety performance successfully assessed. Two significant accidental situations were considered: ULOF (Unprotected Loss Of Flow accident) and UTOP (Unprotected Transient Over-Power accident). Based on very preliminary results, the introduction of spherepac fuel would not cause any specific SFR design or safety issue. Therefore, thanks to PELGRIMM a significant step forward has been taken [1] in the fuel qualification long term process, making a future of the efforts in the previous European projects CP-ESFR (2008-2013), F-BRIDGE (2008-2012), ACSEPT (20082012), and FAIRFUELS (2009-2015). Besides, links within PELGRIMM and ASGARD FP-7 projects implemented in parallel have led to bridge the fuel development to the fuel cycle back-end.

\section{Materials' Innovation for Safe and Sustainable Nuclear in Europe (MatISSE)}

The MatISSE project was fully embedded in the EERA JPNM, aimed at building a European integrated research programme on materials innovation for a safe and sustainable nuclear. The selected scientific and technical work was directed to progress in the fields of conventional and advanced nuclear materials, including capability to forecast their behaviour in operation, with emphasis on fuel and structural elements for advanced nuclear systems, reflecting the subprogramme structure of the JPNM at the time of the launch of the project.

In addition, MatISSE included a Coordination and Support Action, focused on allowing the evolution of the JPNM towards a more structured and solid way of working, including (i) networking with public authorities, (ii) harmonisation of best practices and implementation of communication tools and (iii) a common research strategy, appropriate organisation, knowledge management and the organisation of project calls.

The R\&D activities of MatISSE were selected as being relevant for the European Sustainable Nuclear Industrial Initiative (ESNII), applying both experimental and theoretical approaches and organized in seven work packages (WP), each one with specific objectives (WP6 and WP7 were dedicated to dissemination, communication, E\&T and management).

WP1 was dedicated to coordination and support to the JPNM. The efforts made in the different tasks of this WP resulted in various good achievements (e.g. description of work document, vision paper, SRA, pilot projects, crosscutting workshops, memorandum of understanding with the Sustainable Nuclear Energy Technology Platform (SNETP), education and training scheme, JPNM website) and hence further developed the JPNM as integrated research programme.

WP2 was organized in two research areas, one devoted to the modelling of the microstructural embrittling features in irradiated ferrite/martensite $(\mathrm{F} / \mathrm{M})$ alloys and their effect on radiation-induced hardening (MEFISTO), the other to the modelling of irradiation creep starting from its microstructural origin in the same materials (MOIRA). 
Attention was focused on studying the nature, origin and effect of microstructural evolution under irradiation on the induced hardening. Developed atomistic models and dislocation dynamics models lead to determine the effect of the different microstructural features on radiation hardening and resulted in the prediction of the mechanical properties of different steels after irradiation.

WP3 had as objective the characterization of ceramic composites for gas-cooled and lead-cooled fast reactors. This WP focused on the manufacturing and assessment of full ceramic $\mathrm{SiC} / \mathrm{SiC}$, sandwich type $\mathrm{SiC} / \mathrm{SiC}$ (with internal tantalum liner) clad sections and MAX phasebased cermets. Investigations of mechanical, leak tightness and thermal properties of $\mathrm{SiC} / \mathrm{SiC}$ composites were performed and encouraging results on $\mathrm{SiC} / \mathrm{SiC}$ and sandwich clad compatibility with impure flowing He were obtained.

WP4 focused on characterization of oxide dispersion strengthened (ODS) alloys for lead-cooled and sodiumcooled fast reactor cladding. A comprehensive and consistent description of the microstructures and mechanical properties of the ODS alloys extruded bars and tubes was performed, leading to a better understanding of the properties of these materials. $14 \mathrm{Cr}$ ODS tube showed a higher resistance than the 9Cr ODS tube during internal pressure creep tests.

WP5 consisted of four tasks addressing topics that had been identified by the European Sustainable Nuclear Industrial Initiative (ESNII) reactor designers: (i) develop models and conduct mechanical tests for creep-fatigue of $\mathrm{F} / \mathrm{M}$ and austenitic steels with emphasis on cyclic softening and crack propagation; (ii) evaluate the compatibility of some specific designed coatings for claddings and surface alloys for structural materials with $\mathrm{Pb}$ alloys as the working fluids; (iii) investigate fuel-cladding interactions for fuel pin of advanced nuclear systems, providing guidelines to include fuel-cladding interaction in the design; (iv) investigate the mechanisms of crack initiation and propagation under constant and cyclic load conditions for $\mathrm{F} / \mathrm{M}$ steels and austenitic steels in lead based alloys.

\section{GenIV Materials Maturity (GEMMA)}

The GEMMA project addresses research, development, qualification and standardization of austenitic steels for GENIV reactors and technologies, including their protection and welding, this being one of the main research lines identified in the EERA JPNM SRA.

Through a wide use of experimental techniques, the project intends to:

- Qualify existing materials for the hostile conditions that are envisaged in GENIV systems.

- Perform screening for the selection of new materials, expected to be more resistant to the typical conditions encountered in GEN IV applications.

- Develop protective coatings to mitigate the effect of corrosion in GEN IV reactors.

- Improve and validate predictive models of material damage through dedicated experiments and forthcoming model refinement.
Presently, the materials to be qualified, including corrosion-protected materials and welded joints of various kinds, have been developed and distributed to the partners to allow the qualification to start. The base materials are slabs and plates of AISI 316L and 15-15 Ti steels, in both the MYRRHA (prototype accelerator driven system) and ALFRED (prototype lead-cooled fast reactor) variants. The welds were produced by tungsten inert gas (TIG) and Submerged arc welding (SAW) techniques, which were optimized in the project itself. Protections from corrosion were applied using innovative GESA (Gepulste Elektronen Strahl Anlage) methods and both PLD (Pulsed Laser Deposition) and Detonation Gun coatings; protected specimens will be subjected to mechanical and corrosion tests.

Effort was devoted to develop and test Alumina Forming Austenitics (AFA) steels. The most promising ones, in terms of corrosion resistance, were selected through accurate screening of properties, among over twenty different chemical compositions, in particular different aluminum, chromium and reactive element contents, with the contribution of an important European steel-maker. This industrial involvement will enable a rapid shift to large-scale production for the most promising material and subsequent access to market.

Concerning welds, in addition to conventional testing a careful assessment of post-weld residual stresses was carried out on a welded slab that accurately reproduces the welds of the main vessel of ASTRID (prototype sodium-cooled fast reactor) by high resolution neutron diffractometry, a technique that accurately detects even the smallest deformations of the crystalline lattice. This experiment is also aimed to validate stress models developed by GEMMA partners. It should be noted that the neutron diffraction of large welded pieces constitutes a novel application and permits a precise and volumetrically distributed evaluation of the tensional state within the joint. In parallel, thermodynamic and kinetic models for Fe-Ni-Cr model alloys under irradiation were developed; experimental studies of elemental diffusion phenomena on multi-layered samples, produced in the Project, will be used for model validation.

\section{Investigations Supporting MOX Fuel Licensing in ESNII Prototype Reactors (INSPYRE)}

Fuel is an essential component of all nuclear reactor systems. Numerous coupled phenomena are induced in the fuel by nuclear fission, e.g. production of defects, fission product migration and interaction, fission gas bubble precipitation, grain restructuring, swelling, cracking. These have an impact on all fuel properties: physical, chemical, thermal and mechanical. These phenomena also have an intrinsic multiscale character, taking place from the nanometre scale to the fuel element one. Mastering the understanding of fuel behaviour under irradiation is therefore challenging. Fuel performance predictions for licensing under normal operation and accidental conditions have relied traditionally upon 
extensive integral irradiation testing (full length pins and assemblies) to generate empirical laws. Though successfully deployed for the four fast reactors operated in Europe thus far, they are not easily extrapolated to other conditions (high $\mathrm{Pu}$ content, low temperature operation, coolant interactions, etc.) prevalent for the licensing of first MOX (mixed oxides) cores for all four reactor systems of ESNII.

Leveraging the knowledge from past integral irradiation testing programmes is essential to overcome the challenges of timely cost effective licensing of ESNII reactor MOX first cores. The solution lies in a basic science approach to develop the intricate models underpinning the empirically derived performance laws, so that they can be extended into other operational regimes. A first proof of principle of this approach was made on $\mathrm{UO}_{2}$ fuels in the F-BRIDGE project (20082012). ${ }^{12}$ This approach can now be applied to the fuels envisaged for the ESNII prototypes to bring significant advances to the licensing of these fuels.

INSPYRE is the unique path forward to cost effective nuclear fuel licensing, through a thorough understanding of fuel performance and safety issues. The goals of INSPYRE focussed almost exclusively on MOX fuel are:

- To use out of pile separate effect investigations and physical modelling and simulation at various scales to complement the information obtained from PIE on irradiated fuels and get further insight into basic phenomena governing fuel behaviour.

- To perform additional PIE on selected samples to yield currently scarce data.

- To use the improved understanding obtained to derive new models describing the behaviour of fuel under irradiation and extend the reliability regime of current laws, which are mostly empirical.

- To implement the models developed in operational fuel performance codes to improve their reliability and efficacy both in normal and off-normal situations.

INSPYRE is composed of 7 technical WPs:

- Four WPs (1-4) underpin the programme by studying four important operational issues using a basic research approach combining multiscale and thermodynamic modelling and separate effect experiments: margin to fuel melting; atom transport and fission product behaviour; evolution of mechanical properties under irradiation; fuel thermochemistry and fuel-cladding interaction.

- WP5 combines the results of WP 1 to 4 with characterization of neutron-irradiated fuels to determine the elementary mechanisms of fast reactor fuel behaviour under irradiation.

- WP6 uses the results obtained in WP1 to 5 and in other projects to develop improved models describing fuel behaviour.

- WP7 then implements the new models and data in fuel performance codes, benchmarks the new versions of the codes and validates them for conditions relevant to the ESNII prototypes.

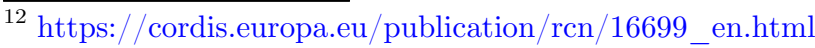

By efficiently leveraging relevant past knowledge and by combining PIE and basic science approaches, within a well-balanced consortium of universities, research and industrial centres, all collaborating within the EERA JPNM, INSPYRE will impact crucially on the extension of the applicability of fuel performance codes, thereby enabling the reduction of the need for integral irradiation test and thus accelerating the licensing procedures, while improving safety standards.

\section{Multiscale Modelling for Fusion and Fission Materials (M4F)}

The main goal of the M4F project is to bring together the fusion and fission materials communities working on the prediction of microstructural-induced radiation damage and deformation mechanisms of irradiated $\mathrm{F} / \mathrm{M}$ steels, which are candidate structural materials in both GenIV fission and fusion reactors. The M4F project is multidisciplinary in nature and integrates models and experiments at different scales to foster the understanding of the complex physical phenomena associated with the formation and evolution of irradiation induced defects and their role on the macroscopic mechanical properties, particularly deformation behaviour.

Specifically, the project focuses on three interrelated issues, each of them requiring intense model development and dedicated experimental support:

- Describe as accurately as possible, through computational physical models, the microstructure evolution under neutron irradiation of $\mathrm{F} / \mathrm{M}$ steels, taking into account simultaneously (i) the influence of the magnetic properties of the $\mathrm{Fe}-\mathrm{Cr}$ system and the redistribution of Cr under irradiation (segregation and precipitation), (ii) the effect of $\mathrm{C}$ and (iii) the role of minor solutes such as Mn, Ni, Si, P. The models should allow the density, size distribution and chemical composition of the radiation-induced features that produce hardening to be predicted, at least up to a few dpa.

- Taking into account the microstructure induced by irradiation, develop meso-scale and continuum scale models, to describe plastic flow localization (i.e. localized deformation with loss of elongation in a tensile test) in F/M steels, at the level of single grain and then in polycrystals, through the elaboration of suitable homogenization methods and physically-based constitutive equations. The models should eventually allow the role of slip localization after irradiation on the mechanical behaviour of loaded components to be quantitatively assessed, so that design criteria can be derived.

- Develop a methodology to design and perform ion irradiation experiments as "surrogate" of neutron irradiation, with control on the potential artifacts that can be encountered in this type of irradiation, and to extract information not only on microstructural changes but also on the corresponding mechanical response, by means of nanoindentation. This requires on the one hand to develop microstructure evolution modeling tools with features suitable to simulate ion irradiation, particularly 
to account for damage gradients along the full ion penetration path and the closeness of a free surface; and, on the other, to establish best practice guidelines and possibly standards to perform nanoindentation measurements, being aware of which type of properties can be realistically deduced from them.

A side objective is to promote interaction and exchange between the fission and fusion materials scientific communities, in order to foster collaboration and to create the framework for future cross-cutting projects.

The project is accordingly structured in three domains (DM): DM1-irradiated microstructure; DM2-plastic deformation; DM3-management (including data management, dissemination and fission/fusion interaction). Currently, all the experimental matrices have been established and the experiments, including irradiations, are in due course. Significant advances have been made in the development of all models, although for the moment the results of their application are limited.

\section{TRANSversal Actions for Tritium (TRANSAT)}

The management of tritium in fusion or fission facilities is based on the development of various key points, which are (i) the implementation of techniques and strategies to mitigate tritium releases, (ii) the control of tritium during the management of tritiated waste, and (iii) the development of new diagnostics to assess the surface and in-depth contamination of this waste. Although tritium is considered to be weakly radiotoxic, no thorough studies exist on the consequences of contamination by tritiated particles resulting from decommissioning operations. It is therefore necessary to improve our knowledge of the radiotoxicity, radiobiology and dosimetry of these particles.

These different topics are part of the major objectives of the TRANSAT programme [2].

From the design stage of a fission reactor, it is essential to plan operations that limit tritium sources to the lowest possible levels. Thus the amount of Boron or Lithium is limited as much as possible.

Similarly, the approach of the fusion community is to optimize the combustion of tritium and its recirculation during the operation of the machine in order to limit its absorption in the walls of the machine or in the tritium plant.

However, the operational constraints do not allow this to go below a certain limit. It is therefore mandatory to develop strategies or techniques to limit the permeation of tritium or its capture in order to limit its release into the environment.

TRANSAT therefore aims to develop technologies to control or reduce the permeation of tritium between and through circuits. This can be achieved, for example, by developing new materials capable of blocking the diffusion of tritium, or by using in-situ treatment of effluents produced during the operation of the machine.
In addition, tritium permeation control also requires knowledge of the tritium inventory and estimation of tritium permeation fluxes in fusion and fission devices. This requires the development of robust modelling tools. In TRANSAT, the modeling tools used in fission and fusion to predict tritium inventory and permeation are compared and calibrated to improve the confidence level in their estimation.

TRANSAT will also work on the evaluation of technological solutions proposed for tritium production and the management of tritiated waste as an important open issue common to both fusion and fission.

Tritium is an isotope of hydrogen and as such is easily absorbed by any material. This results in tritium releases whose magnitude and kinetics are related to the tritium inventory and profile in the samples under consideration.

The strategy for the disposal of tritium-contaminated wastes is complex and is based on open critical issues such as:

- Accurate and robust measurement of tritium in the sample at its surface but also at the core of the material under consideration. Non-destructive techniques have limited accuracy, while destructive methods depend on a sampling strategy that is unsatisfactory due to possible inhomogeneity. However, none of these techniques provide access to information on the tritium profile. In TRANSAT, innovative measurement techniques will be used to assess the tritium inventory and profile.

- The development of mitigation strategies to keep tritium releases below the storage facility acceptance criteria. These include the treatment of tritiated waste (thermal treatment, incineration, etc.), the improvement of the containment drum, the development of containment matrices, etc.

These methods can be combined or used separately. Considering detritiation processes, various research and development activities are already funded at European level under H2020/ Power Plant Physics \& Technology (WP Safety and Environment). As part of the waste management strategy, TRANSAT will therefore focus on improving new containment drum concepts.

In addition, the TRANSAT project will work to improve knowledge in the field of radiobiology, dosimetry, radiotoxicology, genotoxicology, ecotoxicology and the fate in the environment in the event of contamination by tritiated products.

Numerous studies have been carried out on the radiotoxicological consequences of contamination by tritiated water or the Organic Bound Tritium in animals or cells. As a consequence of these studies, tritium in these forms is considered to be weakly radiotoxic. However, during the dismantling of nuclear installations, fine tritiated particles suspended in the air (aerosols) can be created. The consequences of contamination by these tritiated particles have never been studied in terms of radiotoxicology and ecotoxicology. Among TRANSAT's important goals are also these innovative studies. The results will enable the radiation protection authorities, the IAEA and other 
nuclear safety advisory bodies to assess more precisely the radiobiology, dosimetry, genotoxicology and ecotoxicology of tritiated particles of the order of micron and submicron.

\section{Summary and conclusions}

The EERA JPNM provides a consistent framework under which activities related with the qualification, modeling and development of structural and fuel materials for advanced and innovative nuclear systems, towards full nuclear energy sustainability, are coordinated. Substantial contributions from institutional funding effectively integrate the Euratom support: in all projects under this umbrella, including those belonging to H2020, the total budget significantly exceeds the Euratom contribution, thanks to the fact that these projects are the result of an alignment between national programmes that preceded their launch, i.e. they are based on JPNM pilot projects that are suitably combined to fit the calls. Even though PELGRIMM preceded the inclusion of fuel activities in the JPNM, it follows similar philosophy in terms of approach and topics. All this provides a strong basis to build, in the near future, an efficient European Joint Programme (EJP) on nuclear materials, within which Members States and European Commission earmark funding specifically devoted to this crucial topic. Materials are indeed key for all nuclear reactor generation safety, economy and sustainability, including fusion systems, and also offer the possibility of establishing links with other low-carbon energy technologies, particularly within EERA. Because of the harsh operating conditions and strict safety rules it has to comply with, the expertise on materials for the nuclear field can indeed produce spin-offs that are applicable to other energy systems where extreme operating conditions are faced.

The projects described in this paper also provide a couple of successful examples of cross-cutting actions between fission and fusion. These certainly represent a formula to be pursued more intensively in the future, because of the mutual benefit that cross-fertilization always brings, and especially because this formula, applied to properly identified topics, ensures that an optimal use of human and infrastructural resources is made, without costly duplications.

\section{References}

1. A. Gallais-During, F. Delage et al., Outcomes of the PELGRIMM project: progress in the development of Ambearing fuel under pelletized and spherepac forms, J. Nucl. Mat. 512, 214 (2018)

2. K. Liger, C. Grisolia, I. Cristescu, C. Moreno, V. Malard, D. Coomb, S. Markelj, Overview of the TRANSAT (TRANSversal Actions for Tritium) project, Fusion Eng. Des. 136, 168 (2018)

Cite this article as: Lorenzo Malerba, Pietro Agostini, Marjorie Bertolus, Fabienne Delage, Annelise Gallais-During, Christian Grisolia, Karine Liger, Pierre-François Giroux, Advances on GenIV structural and fuel materials and cross-cutting activities between fission and fusion, EPJ Nuclear Sci. Technol. 6, 32 (2020) 